\title{
Correlated phases for fermions on a Kagome lattice
}

\author{
Discovery of unconventional chiral charge order in kagome superconductor \\ $K V_{3} S b_{5}$ \\ Authors: Yu-Xiao Jiang et al \\ arXiv: 2012.15709
}

\section{Recommended with a Commentary by Rahul Nandkishore, University of Colorado Boulder}

New material platforms have a history of opening new chapters in condensed matter physics, with cuprates, pnictides, and Moire heterostructures providing some of the most storied examples. Recently a new class of materials has entered the fray. These are the quasi-two dimensional, exfoliable materials $A V_{3} S b_{3}$, where $A$ is an alkali atom $(K, C s, R b)$. Each layer consists of a Kagome net of $V$ atoms coordinated by $S b$, and with additional $S b$ and alkali atoms sandwiched between Kagome layers. The structure is illustrated in Fig. 1a.

In the past six months these materials have been the subject of a host of papers both experimental (the featured paper, and also $[1,2,3,4,5,6,9,7,8]$ ) and theoretical $[10,11,12$, $13,14]$ (this is not a complete list). The non-interacting (high temperature) bandstructure has been calculated in DFT and measured in ARPES - an idealized version of the Fermi surface that is believed to obtain is shown in Fig.1b. It consists of a hexagon inscribed within the Brillouin zone, plus additional Fermi pockets at the $\Gamma$ point and on the $\Gamma M$ line. The inscribed hexagon has two special properties - it displays a high degree of nesting, and the corners of the hexagon (the $M$ points) are saddle points of the dispersion giving rise to a (logarithmic) Van Hove singularity in the density of states. Both the nesting and the Van Hove singularity are expected to greatly favor formation of correlated states, and indeed interaction driven instabilities of the hexagonal Fermi surface have been extensively discussed in the theory literature $[15,17,16]$, and a multitude of states including chiral superconductors [15] and chiral insulators [16] have been proposed. Now, finally, experiments can explore this scenario.

Experiments indeed reveal a cornucopia of ordered phases. A complete survey of the experimental literature is beyond the scope of this commentary, but I will summarize below what I see as the key results. All these materials $(\mathrm{A}=K, C s, R b)$ exhibit charge density wave (CDW) order at a critical temperature in the range around $80-100 K$, and superconductivity (SC) with a critical temperature on the order of $1 K$. Scanning tunneling microscopy (STM) measurements and X-ray spectroscopy indicate that the CDW order is 'hexagonal,' with ordering at three distinct wavevectors (Fig. 2a), and with the ordering wavevectors being equal to the nesting vectors of the hexagonal Fermi surface. In addition, the three wavevector 
(a)

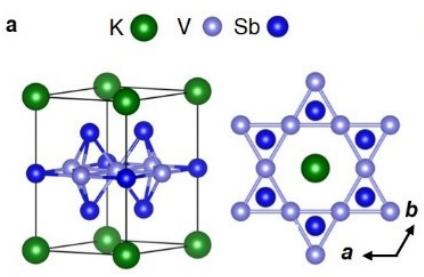

(b)

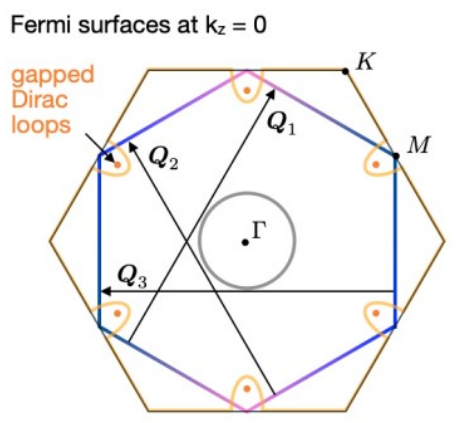

Figure 1: (a) An illustration of the material structure of $A V_{3} S b_{3}$. The low energy electron states are believed to live largely on the $V$ atoms. (b) An illustration of the idealized Fermi surface. The largest Fermi pocket consists of a single hexagon inscribed within the Brillouin zone. The corners of the hexagon (M points) are saddle points of the dispersion and give rise to a logarithmic divergence in the density of states (Van Hove sigularity). There are three inequivalent $M$ points. In addition, there are Fermi pockets at the $\Gamma$ point and a small Fermi pocket on the line $\Gamma M$, which comes from a Dirac point. Figures are taken from arXiv: 2012.15709, with permission

peaks have an intensity anisotropy which is argued to be chiral. The system appears to have a quadrupled in plane unit cell. There is large (but not quantized) anomalous Hall effect in the CDW regime, but no sign of local moments in muon spin spectroscopy. The CDW state appears to feature unusual magnetic tuneability, also suggesting chirality and raising tantalizing connections to classic theory works on chiral insulators with orbital currents $[18,19]$.

Some key questions raise themselves. The first set involves completing the experimental characterization of the system. What symmetries are broken in the CDW state? The presence of a large anomalous Hall effect and the switch-ability by magnetic field suggest time reversal symmetry breaking, but muon spin spectroscopy does not see local moments. Can we directly confirm if the CDW state itself breaks time reversal symmetry? Meanwhile, what is the nature of the anisotropy? Chiral (as argued), nematic, or something else? Is it there throughout the CDW phase, or is the chiral/nematic transition separate from the CDW transition, such that there are in fact two CDW phases? Furthermore, given that these are layered materials, what is the three dimensional (i.e. inter-layer) structure of the CDW? Meanwhile, superconductivity develops around 1K. Does CDW order co-exist with superconductivity, or does it disappear at the superconducting transition? What is the symmetry of the superconducting state? How does the phase structure change under doping? Under pressure? Under applied magnetic field? Are there any additional phases besides the CDW and superconductor? What does the full phase diagram look like?

A parallel set of questions involves the theoretical understanding of what is going on. The nested 'M point' Fermi surface offers a natural route to correlated phases that exploits both the nesting and the divergent density of states. This approach begins by parametrizing electron electron interactions in terms of a finite number of ' $g$ ' parameters (g-ology - see Fig.2b), and then examines the leading weak coupling instability within a perturbative renormalization group perspective. This approach looks much the same on any hexagonal lattice, except 


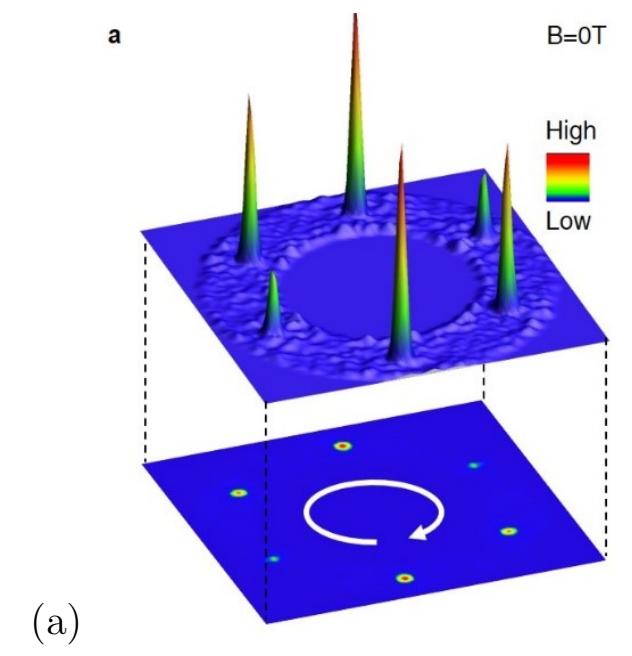

(b)
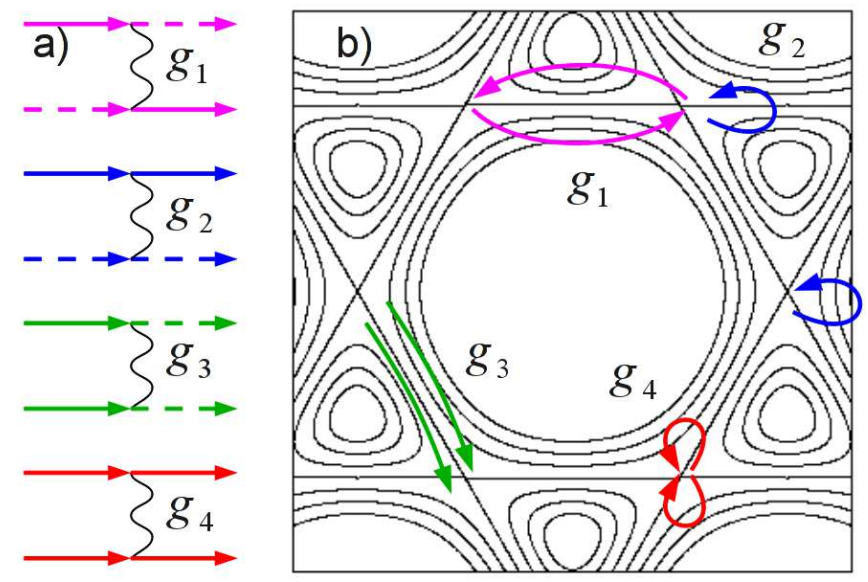

Figure 2: (a) STM measurements on the CDW phase at $4.2 \mathrm{~K}$ reveal ordering at three distinct wavevectors ( $3 Q$ order), with the ordering vectors being equal to the 'nesting vector' for the hexagonal Fermi surface. The strength of ordering appears to exhibit a chiral anisotropy. Figure taken from arXiv: 2012.15709, with permission. (b) The 'g-ology' model for interaction effects on the hexagonal Fermi surface. One reduces the Fermi surface to three 'patches' at the three inequivalent saddle points, and then parametrizes the interactions in terms of four $g$ parameters, shown above, corresponding to inter-patch and intra-patch scattering as appropriate. Figure taken from [15].

that 'sublattice interference' effects on the Kagome lattice [17] can alter the 'bare' (ultraviolet scale) g-parameters. Of course, while the 'g-ology' approach is extremely appealing from a theoretical perspective, it is controlled only at asymptotically weak coupling, and it remains to be seen whether this is an appropriate description for the experimental systems.

More broadly, open theoretical questions include the following: What is the mechanism giving rise to the various phases? If the CDW phase is chiral, is it related to the classic theoretical discussions of phases with orbital currents [18, 19]? Is it related to the observed anomalous Hall effect in twisted bilayer graphene? How does the superconductivity develop inside the CDW phase? Does it have the same mechanism as the CDW phase, or distinct? Can the onset of superconductivity be understood starting from the 'bare' bandstructure, or is reconstruction of the bands by the CDW essential to the onset of superconductivity? What is the interplay of superconducting, CDW and (perhaps) nematic order? Can we predict theoretically what the phase diagram should be, particularly in the presence of applied field, or applied pressure? Should we expect there to be additional phases? And can we usefully guide the experimental exploration of these materials?

As should be clear from the above, much remains to be understood. However, the existing experiments already make clear that there is treasure to be found here, and the rapid development of experiments promises answers to several key questions in the near future. There are exciting times ahead. 


\section{References}

[1] N. Shumiya et al, arXiv: 2105.00550

[2] Hong Li et al, arXiv: 2104.08209

[3] He Zhao et al, arXiv: 2103.03118

[4] Brendan R. Ortiz et al, arXiv: 2012.09097

[5] Kosuke Nakayama et al, arXiv: 2104.08042

[6] Zhengguo Wang et al, arXiv: 2104.05556

[7] Zhiwei Wang et al, arXiv: 2105.04542

[8] E.M. Kenney et al, arXiv: 2012.04737

[9] F.H. Yu et al, arXiv: 2102.10987

[10] T. Park, M. Ye and L. Balents, arXiv: 2104.08425

[11] X. Feng, K. Jiang, Z. Wang, and J. Hu, arXiv:2103.07097

[12] Y.-P. Lin and R. M. Nandkishore, arXiv: 2104.02725

[13] M. M. Denner, R. Thomale, and T. Neupert, arXiv:2103.14045

[14] Y.-P. Lin, arXiv: 2106.09717

[15] R. Nandkishore, L.S. Levitov and A.V. Chubukov Nature Physics 8, 158-163 (2012)

[16] Y.-P. Lin and R. M. Nandkishore, Phys. Rev. B 100, 085136 (2019)

[17] M.L. Kiesel, C. Platt and R. Thomale, Phys. Rev. Lett. 110, 126405 (2013)

[18] F.D.M. Haldane, Phys. Rev. Lett. 61, 2015 (1988)

[19] C. M. Varma, Phys. Rev. B 55, 14554 (1997) 